\title{
Diffusion-weighted MRI in acute posterior ischemic optic neuropathy
}

\author{
Sivasubramanian Srinivasan, Srikant Moorthy'1, KP Sreekumar', Chinmay Kulkarni \\ Department of Diagnostic Radiology, Khoo Teck Puat Hospital, 90, Yishun Cetral, Singapore 768828, 'Department of Radiology, \\ Amrita Institute of Medical Sciences, Elamakkara, Cochin 682041, India \\ Correspondence: Dr. Sivasubramanian Srinivasan, Department of Diagnostic Radiology, Khoo Teck Puat Hospital, 90, Yishun Cetral, \\ Singapore 768828. E-mail: sivasu2@gmail.com
}

\section{Abstract}

Blindness following surgery, especially cardiac surgery, has been reported sporadically, the most common cause being ischemic optic neuropathy. The role of MRI in the diagnosis of this condition is not well established. We present a case of postoperative posterior ischemic optic neuropathy that was diagnosed on diffusion-weighted MRI.

Key words: Postoperative vision loss; ischemic optic neuropathy; diffusion-weighted MR imaging

\section{Introduction}

Perioperative vision $\operatorname{loss}^{[1,2]}$ due to ischemic optic neuropathy may present as sudden blindness after surgeries such as cardiac surgery, head and neck surgeries, spine surgeries and major abdominal surgeries. ${ }^{[3]}$ Anterior ischemic optic neuropathy is easily diagnosed by fundoscopic findings, but posterior ischemic optic neuropathy (PION) is difficult to diagnose. ${ }^{[1]}$

Postoperative PION may occur without any other ischemic effect in the eye or central nervous system ${ }^{[1]}$ and may be unilateral or bilateral. In cases of bilateral PION, a high index of suspicion is necessary for diagnosis as the optic nerves initially appear normal and no afferent pupillary defect may be evident. ${ }^{[2]}$

The utility of imaging in ischemic optic neuropathy was not known until recently. ${ }^{[3,4]}$ We report a case of PION that was diagnosed by diffusion-weighted MRI.

\begin{tabular}{|l|l|}
\hline \multicolumn{2}{|c|}{ Access this article online } \\
\hline Quick Response Code: & \\
\hline & Website: \\
\hline & www.ijri.org \\
\cline { 2 - 3 } & DOI: \\
\hline
\end{tabular}

A 50-year-old man presented to the hospital with sudden loss of vision in his right eye, followed by loss of vision in the left eye on the next day. He had undergone coronary bypass surgery and mitral valve replacement 15 days before the presentation, and the immediate postoperative period had been uneventful.

MRI was performed with a $1.5 \mathrm{~T}$ Siemens Symphony ${ }^{\circledR}$ MRI scanner (Siemens Erlangen, Germany). A standard quadrature coil (head coil) was used. The section thickness for diffusion-weighted MRI was $5 \mathrm{~mm}$, with $0.5 \mathrm{~mm}$ gap, matrix: $128 \times 128$, and field of view: $30 \mathrm{~cm}$. MRI of the brain was normal. However, it showed symmetric high signal intensity in the optic nerves on diffusion imaging [Figure 1A], with decreased apparent diffusion coefficient (ADC) [Figure 1B], indicating acute ischemic injury/ infarction. The ADC was low (25-35) in both optic nerves. The anterior-most part of the optic nerves, close to fundus, did not show diffusion restriction. Conventional spin-echo sequences were unremarkable [Figure 1C].

The patient was managed conservatively but he did not regain vision even after 2 months.

\section{Discussion}

Vision loss due to ischemia can be broadly classified ${ }^{[1]}$ into anterior ischemic optic neuropathy and posterior ischemic optic neuropathy as the blood supply to the anterior part 


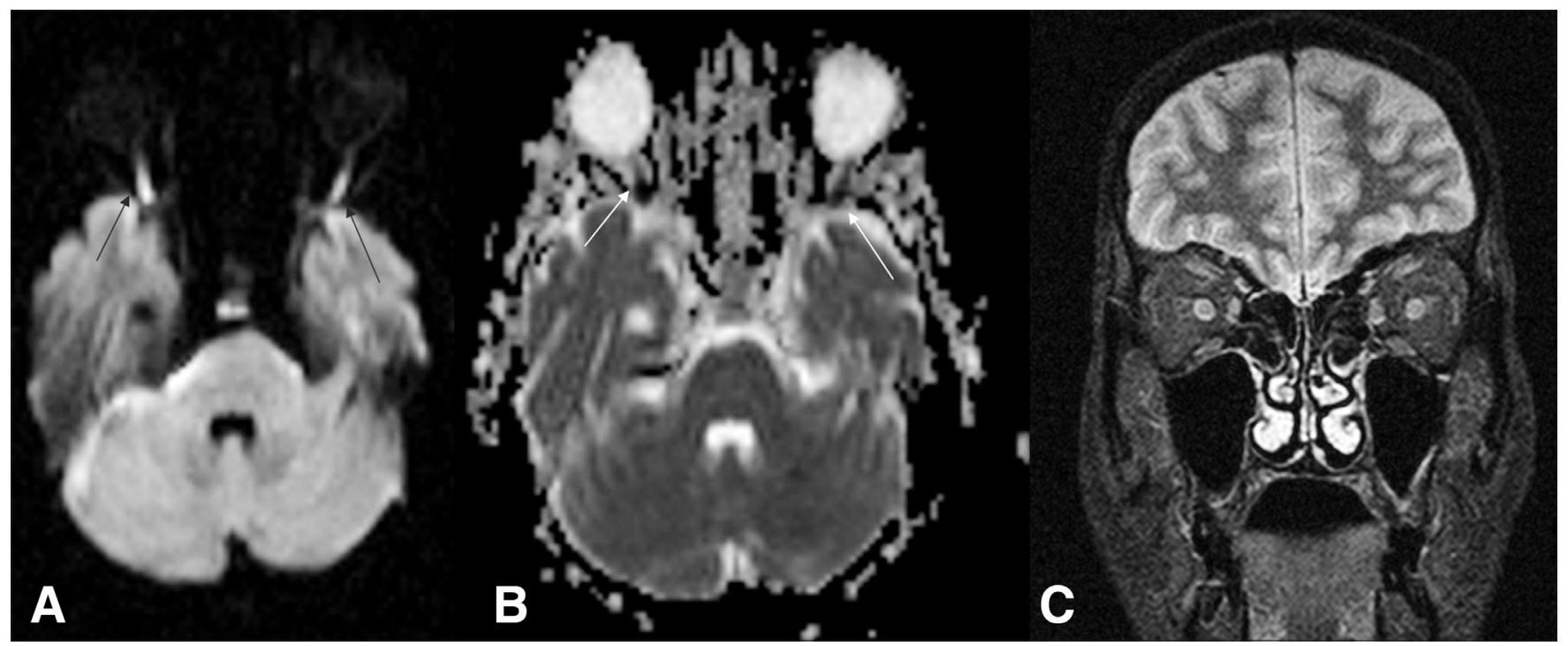

Figure 1 (A-C): Diffusion-weighted (b1000) MRI (A) shows hyperintense signal in the optic nerves (arrows). ADC (apparent diffusion coefficient) image (B) shows restricted diffusion (arrows) in the optic nerves. STIR-coronal image (C) of the orbits posterior to the globes shows normal optic nerves

and posterior part of the optic nerves is different and as the mechanism of ischemia is also different.

The anterior portion of the optic nerve, which includes the optic disc and the small part of the optic nerve within the scleral canal, is supplied mainly by the short posterior ciliary arteries via the choriocapillaries around the optic disk or through branches that form an anastomotic microvascular ring around the optic nerve. Rarely, there is a double arterial supply, with pial branches of the ophthalmic artery also providing blood supply to this section of the optic nerve. ${ }^{[1]}$ Because of this rich blood, this part is less prone to ischemia.

The posterior portion of the optic nerve has a peripheral vascular supply from pial branches of the ophthalmic artery. The central retinal artery often supplies branches to the central fibers. However, the blood flow in the posterior optic nerve is significantly lower than that in the anterior portion.

Hence, this part of the optic nerve (i.e., the segment between the optic foramen and the the point where the central retinal artery enters the nerve) is relatively more susceptible to ischemia during the postoperative period, as the only supply is through centripetal pial vessels that are easily compressible.

Posterior optic nerve ischemia ${ }^{[1,2]}$ however is less common than anterior ischemic optic neuropathy. PION has been reported after various surgeries like laparotomy and, cardiac, neck and spinal surgeries. ${ }^{[2,3]}$ The exact cause is not clear; proposed theories include severe hypotension and anemia and, rarely, other factors like infection, congenital absence of central retinal artery, venous occlusion, etc.

Only five reports of MRI findings of ischemic optic neuropathy ${ }^{[3-6]}$ could be found in the literature after an extensive search. Our patient is the only the second case to be reported of postoperative/perioperative $\mathrm{PION}^{[4]}$ showing restricted diffusion. Other reported cases of ischemic optic neuropathy with restricted diffusion were due to mucormycosis. ${ }^{[5,6]}$

\section{Conclusion}

Radiologists should be aware of this entity and attention should be paid to the diffusion-weighted images, where the ischemic or infarcted optic nerve shows restricted diffusion. Clinicians should be encouraged to refer patients for MRI if PION is suspected.

\section{References}

1. Williams EL, Hart WM Jr, Tempelhoff R. Postoperative ischemic optic neuropathy. Anesth Analg 1995;80:1018-29.

2. Murph MA. Bilateral posterior ischemic optic neuropathy after lumbar spine surgery. Ophthalmology 2003;110:1454-7.

3. Park JY, Lee IH, Song CJ, Hwang HY. Diffusion MR Imaging of Postoperative Bilateral Acute Ischemic Optic Neuropathy. Korean J Radiol 2012;13:237-9

4. Purvin V, Kuzma B. Intraorbital optic nerve signal hyperintensity on magnetic resonance imaging sequences in perioperative hypotensive ischemic optic neuropathy. J Neuroophthalmol 2005;25:202-04.

5. Al-Shafai LS, Mikulis DJ. Diffusion MR imaging in a case of acute ischemic optic neuropathy. AJNR Am J Neuroradiol 2006;27:255-7.

6. Mathur S, Karimi A, Mafee MF. Acute optic nerve infarction demonstrated by diffusion-weighted imaging in a case of rhinocerebral mucormycosis. AJNR Am J Neuroradiol 2007;28: 489-90.

Cite this article as: Srinivasan S, Moorthy S, Sreekumar KP, Kulkarni C. Diffusion-weighted MRI in acute posterior ischemic optic neuropathy. Indian J Radiol Imaging 2012;22:106-7.

Source of Support: Nil, Conflict of Interest: None declared. 